Gazi University
Journal of Science
PART A: ENGINEERING AND INNOVATION
http://dergipark.org.tr/gujsa

\title{
Investigating (p,q)-hybrid Durrmeyer-type Operators in terms of Their Approximation Properties
}

\author{
Ülkü DINLEMEZ KANTAR ${ }^{* *}$, İsmet YÜKSEL ${ }^{1(i D}$
}

${ }^{I}$ Gazi University, Faculty of Science, Department of Mathematics, Ankara/TURKEY

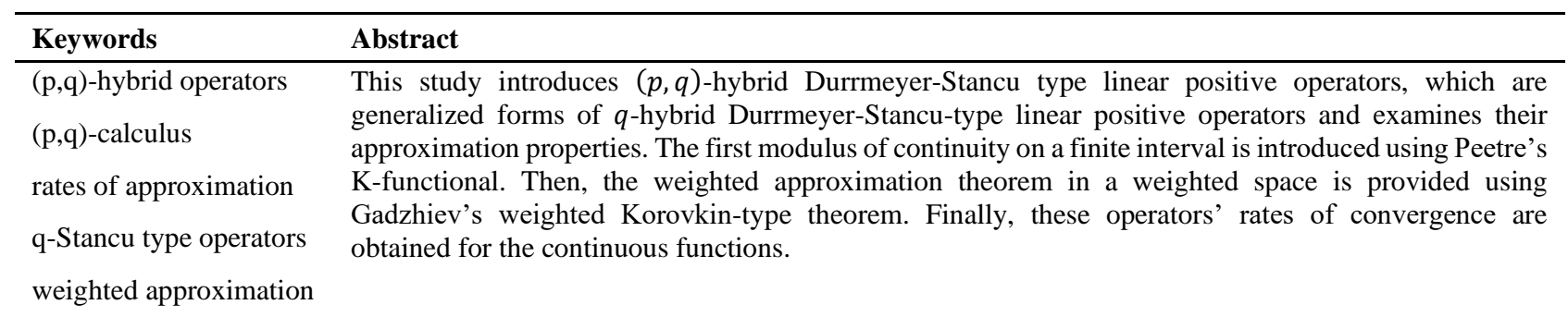

\section{Cite}

Dinlemez Kantar, U., \& Yuksel, I. (2022). Investigating (p,q)-hybrid Durrmeyer-type operators in terms of their approximation properties. GU J Sci, Part A, 9(1), 1-11.

\begin{tabular}{lrl}
\hline Author ID (ORCID Number) & Article Process \\
\hline Ü. Dinlemez Kantar, 0000-0002-5656-3924 & Submission Date & 28.11 .2021 \\
İ. Yüksel, 0000-0002-2631-2382 & Revision Date & 04.01 .2022 \\
& Accepted Date & 19.01 .2022 \\
& Published Date & 25.01 .2022 \\
\hline
\end{tabular}

\section{INTRODUCTION}

In Dinlemez et al. (2014), they introduced $q$-hybrid Durrmeyer-Stancu type linear positive operators for $0<q \leq 1$ as

$$
H_{m, q}^{\alpha, \beta}(g, x)=\sum_{k=1}^{\infty} s_{m, k, q}(x) \int_{0}^{\infty / A} b_{m, k-1, q}(t) g\left(\frac{[m]_{q} t+\alpha}{[m]_{q}+\beta}\right) d_{q} t+e^{-[m]_{q} x} g\left(\frac{\alpha}{[m]_{q}+\beta}\right),
$$

where

$$
s_{m, k, q}(x)=\frac{e^{-[m]_{q} x}[m-1]_{q}}{[k]_{q} !}\left([m]_{q} x\right)^{k}
$$

and

$$
b_{m, k, q}(x)=\left[\begin{array}{c}
m+k-1 \\
k
\end{array}\right]_{q} q^{k(k-1)} \frac{x^{k}}{(1+x)_{q}^{m+k}} .
$$

are $q$-Szász and $q$-Baskakov basis functions, respectively. A $q$-analogue of the Bernstein operators was introduced by Lupaş (1987). These operators were based on $q$-integer and $q$-binomial coefficients for the first time. Then, a number of interesting generalizations about q-calculus were studied by Jackson (1910), Koelink \& Koornwinder (1990), Phillips (1997), Kac \& Cheung (2002), De Sole \& Kac (2005), Doğru \& Gupta (2005, 2006), Gupta \& Heping (2008), Gupta \& Aral (2010), Gupta \& Karsli (2012), Aral et al. (2013), Yüksel (2013). 
Sahai \& Yadav (2007), Kanat \& Sofyalığlu (2018), Sofyalığlu et al. (2021) introduced the generalization of $(p, q)$-calculus. Recently, the series of studies on $(p, q)$-generalizations with a sequence of linear positive operators have been made by Mursaleen et al. (2015 a,b,c), Acar et al. (2016, 2018), Gupta (2018), Cai et al. (2021), Kanat \& Sofyalığlu (2021). Our objective is going to obtain the generalization of $(p, q)-$ calculus of hybrid Durrmeyer-Stancu type operators in Dinlemez et al. (2014).

\section{PRELIMINARIES AND NOTATIONS}

Some basic formulas in $(p, q)$-calculus in the literature can be obtained using basic $q$-calculus as follows

$$
\begin{array}{cc}
{[m]_{p, q}=\frac{p^{m}-q^{m}}{p-q},} & {[m]_{p, q} !=[1]_{p, q}[2]_{p, q} \ldots[m]_{p, q},} \\
(a \oplus b)_{p, q}^{m}=(a+b)(a p+b q)\left(a p^{2}-b q^{2}\right) \ldots\left(a p^{m-1}-b q^{m-1}\right), & {[m]_{p, q}=p^{m-1}[m]_{q / p},} \\
d_{p, q} f(x)=f(p x)-f(q x), & (a \oplus b)_{p, q}^{m}=p^{\frac{m(m-1)}{2}}(a+b)_{q / p}^{m} . \\
{[m]_{p, q} !=p^{\frac{m(m-1)}{2}[m]_{q / p} !,}}
\end{array}
$$

We define the $(p, q)$-beta functions $B_{p, q}(k, m)$ as follows

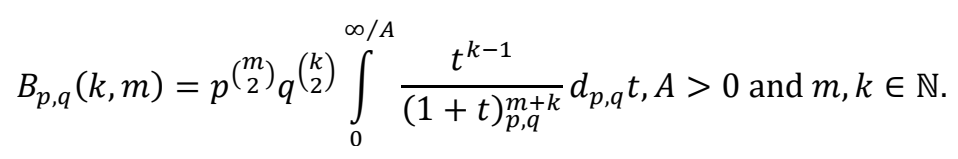

\section{3. $(p, q)$-HYBRID OPERATORS}

Let $A>0, k \in \mathbb{N}, m \in \mathbb{N} \backslash\{0\}$, and $f$ is a continuous function with real-value in the interval $[0, \infty)$. Then, $(p, q)$ - hybrid Durrmeyer-Stancu type linear positive operators are written for $0<q<p \leq 1$ as follows

$$
H_{m, p, q}^{\alpha, \beta}(g, x)=\sum_{k=1}^{\infty} s_{m, k, p, q}(x) \gamma_{m, k}(p, q) \int_{0}^{\infty / A} b_{m, k-1, p, q}(t) g\left(\frac{p^{-m}[m]_{p, q} t+\alpha}{[m]_{p, q}+\beta}\right) d_{p, q} t+e^{-[m]_{p, q} x} g\left(\frac{\alpha}{[m]_{p, q}+\beta}\right),
$$

where

$$
\begin{gathered}
s_{m, k, p, q}(x)=\frac{e^{-[m]_{p, q} x}[m-1]_{p, q}}{[k]_{p, q} !}\left([m]_{p, q} x\right)^{k}, \\
b_{m, k, p, q}(x)=\left[\begin{array}{c}
m+k-1 \\
k
\end{array}\right]_{p, q} \frac{x^{k}}{(1+x)_{p, q}^{m+k}},
\end{gathered}
$$

and

$$
\gamma_{m, k}(p, q)=q^{k(k-1)} p^{\left(\begin{array}{c}
m-1 \\
2
\end{array}\right)} .
$$

When we set $p=1$ in (3), the operators $H_{m, p, q}^{\alpha, \beta}$ are reduced to $q$-hybrid Durrmeyer-Stancu type operators given in (1). Along with the manuscripts, the following notations will be used

$$
R_{p, q}(m, \beta)=\left([m]_{p, q}+\beta\right), \quad T_{p, q}(m, s)=\prod_{i=2}^{s}[m-i]_{p, q} .
$$

And now the lemma for the Korovkin test functions can be given as follows: 
Lemma1 When $e_{r}(t)=t^{r}, r=0,1,2$, we get

(i) $\quad H_{m, p, q}^{\alpha, \beta}\left(e_{0}, x\right)=1$,

(ii) $\quad H_{m, p, q}^{\alpha, \beta}\left(e_{1}, x\right)=\frac{p^{-2}[m]_{p, q}^{2}}{q R_{p, q}(m, \beta) T_{p, q}(m, 2)} x+\frac{\alpha}{R_{p, q}(m, \beta)^{\prime}}$

(iii) $\quad H_{m, p, q}^{\alpha, \beta}\left(e_{2}, x\right)=\frac{p^{-3}[m]_{p, q}^{4}}{q^{4}\left(R_{p, q}(m, \beta)\right)^{2} T_{p, q}(m, 3)} x^{2}+\left\{\frac{p^{-5}[2]_{p, q}[m]_{p, q}^{3}}{q^{3}\left(R_{p, q}(m, \beta)\right)^{2} T_{p, q}(m, 3)}+\frac{2 \alpha p^{-3}[m]_{p, q}^{2}}{q\left(R_{p, q}(m, \beta)\right)^{2} T_{p, q}(m, 2)}\right\} x+\frac{\alpha^{2}}{\left(R_{p, q}(m, \beta)\right)^{2}}$

Proof After $(p, q)$ - beta functions in (2) are used, it is obtained as follows

$$
\begin{aligned}
\int_{0}^{\infty / A} b_{m, k-1, p, q}(t) t^{r} d_{p, q} t & =\left[\begin{array}{c}
m+k-2 \\
k-1
\end{array}\right]_{p, q} \int_{0}^{\infty / A} \frac{t^{k+r-1}}{(1+t)_{p, q}^{m+k-1}} d_{p, q} t \\
& =\frac{\left.[k+r-1]_{p, q} ![m-r-2]_{p, q} ! q^{-(k+r} \begin{array}{c}
(m) \\
2
\end{array}\right)}{[m-1]_{p, q} ![k-1]_{p, q} ! p\left(\begin{array}{c}
m-r-1 \\
2
\end{array}\right)} .
\end{aligned}
$$

Then, by using (4) for $r=0$, we obtain

$$
\begin{aligned}
H_{m, p, q}^{\alpha, \beta}\left(e_{0}, x\right) & =\sum_{k=1}^{\infty} s_{m, k, p, q}(x) \gamma_{m, k}(p, q) \int_{0}^{\infty / A} b_{m, k-1, p, q}(t) d_{p, q} t+e^{-[m]_{p, q} x} \\
& =e^{-[m]_{p, q} x} \sum_{k=0}^{\infty} \frac{\left([m]_{p, q} x\right)^{k}}{[k]_{p, q} !} q^{-k(k-1) / 2} \\
& =e^{-[m]_{p, q} x} E_{p, q}^{[m]_{p, q} x}=1,
\end{aligned}
$$

and the proof of (i) is completed. The following (ii) is obtained by a direct computation

$$
\begin{aligned}
H_{m, p, q}^{\alpha, \beta}\left(e_{1}, x\right) & =\sum_{k=1}^{\infty} s_{m, k, p, q}(x) \gamma_{m, k}(p, q) \int_{0}^{\infty / A} b_{m, k-1, p, q}(t) \frac{p^{-m}[m]_{p, q} t+\alpha}{R_{p, q}(m, \beta)} d_{p, q} t+\frac{\alpha e^{-[m]_{p, q} x}}{R_{p, q}(m, \beta)} \\
& =\frac{p^{-m}[m]_{p, q}}{R_{p, q}(m, \beta) T_{p, q}(m, 2)} \sum_{k=1}^{\infty} \frac{\left([m]_{p, q} x\right)^{k}}{[k-1]_{p, q} !} q^{k(k-3) / 2} p^{m-3} e^{-[m]_{p, q} x} \\
& +\frac{\alpha}{R_{p, q}(m, \beta)} \sum_{k=1}^{\infty} \frac{\left([m]_{p, q} x\right)^{k}}{[k]_{p, q} !} q^{k(k-1) / 2} e^{-[m]_{p, q} x}+\frac{\alpha e^{-[m]_{p, q} x}}{R_{p, q}(m, \beta)} \\
& =\frac{p^{-2}[m]_{p, q}^{2}}{q R_{p, q}(m, \beta) T_{p, q}(m, 2)} x+\frac{\alpha}{R_{p, q}(m, \beta)}
\end{aligned}
$$

Using the following equality

$$
[s]_{p, q}=q^{s-r}[r]_{p, q}+p^{r}[s-r]_{p, q}, \quad 0 \leq r \leq s,
$$

we get 


$$
\begin{aligned}
H_{m, p, q}^{\alpha, \beta}\left(e_{2}, x\right) & =\sum_{k=1}^{\infty} s_{m, k, p, q}(x) \gamma_{m, k}(p, q) \int_{0}^{\infty / A} b_{m, k-1, p, q}(t)\left(\frac{p^{-m}[m]_{p, q} t+\alpha}{R_{p, q}(m, \beta)}\right)^{2} d_{p, q} t+\frac{\alpha^{2} e^{-[m]_{p, q} x}}{\left(R_{p, q}(m, \beta)\right)^{2}} \\
& =\frac{p^{-2 m}\left([m]_{p, q}\right)^{2}}{\left(R_{p, q}(m, \beta)\right)^{2}} \sum_{k=1}^{\infty} s_{m, k, p, q}(x) \gamma_{m, k}(p, q) \int_{0}^{\infty / A} b_{m, k-1, p, q}(t) t^{2} d_{p, q} t \\
& +\frac{2 \alpha p^{-m}[m]_{p, q}}{\left(R_{p, q}(m, \beta)\right)^{2}} \sum_{k=1}^{\infty} s_{m, k, p, q}(x) \gamma_{m, k}(p, q) \int_{0}^{\infty} b_{m, k-1, p, q}(t) t d_{p, q} t \\
& +\frac{\alpha^{2}}{\left(R_{p, q}(m, \beta)\right)^{2}} \sum_{k=1}^{\infty} s_{m, k, p, q}(x) \gamma_{m, k}(p, q) \int_{0}^{\infty} b_{m, k-1, p, q}(t) d_{p, q} t \\
& +\frac{\alpha^{2} e^{-[m]_{p, q} x}}{\left(R_{p, q}(m, \beta)\right)^{2}} \\
& =\frac{p^{-3}[m]_{, q}^{4}}{q^{4}\left(R_{p, q}(m, \beta)\right)^{2} T_{p, q}(m, 3)} x^{2} \\
& +\left\{\frac{p^{-5}[2]_{p, q}[m]_{p, q}^{3}}{q^{3}\left(R_{p, q}(m, \beta)\right)^{2} T_{p, q}(m, 3)}+\frac{2 \alpha p^{-3}[m]_{p, q}^{2}}{q\left(R_{p, q}(m, \beta)\right)^{2} T_{p, q}(m, 2)}\right\} x+\frac{\alpha^{2}}{\left(R_{p, q}(m, \beta)\right)^{2}} .
\end{aligned}
$$

Thus the proof of (iii) is completed.

For the main results of the study, we need to compute the second moment.

Lemma 2 Assuming that $0<q<p \leq 1$ and $m>3$, we obtain the following inequality

$$
H_{m, p, q}^{\alpha, \beta}\left((t-x)^{2}, x\right) \leq\left(\frac{2\left(1-p^{-2} q^{3}\right)}{q^{4}}+\frac{288(\alpha+\beta+1)^{2}[m]_{p, q}}{q^{4} T_{p, q}(m, 3)}\right)\left(x^{2}+x\right)+\frac{\alpha^{2}}{\left(R_{p, q}(m, \beta)\right)^{2}} .
$$

Proof To write the second moment, we use the result of Lemma 1 and the linearity of $H_{m, p, q}^{\alpha, \beta}$ operators;

$$
\begin{aligned}
H_{m, p, q}^{\alpha, \beta}\left((t-x)^{2}, x\right) & =\left\{\frac{p^{-3}[m]_{p, q}^{4}}{q^{4}\left(R_{p, q}(m, \beta)\right)^{2} T_{p, q}(m, 3)}-\frac{2 p^{-2}[m]_{p, q}^{2}}{q R_{p, q}(m, \beta) T_{p, q}(m, 2)}+1\right\} x^{2} \\
& +\left\{\frac{p^{-5}[2]_{p, q}[m]_{p, q}^{3}+2 \alpha q^{2} p^{-3}[m-3]_{p, q}[m]_{p, q}^{2}}{q^{3}\left(R_{p, q}(m, \beta)\right)^{2} T_{p, q}(m, 3)}-\frac{2 \alpha}{R_{p, q}(m, \beta)}\right\} x \\
& +\frac{\alpha^{2}}{\left(R_{p, q}(m, \beta)\right)^{2}} \\
& \leq\left\{\frac{[m]_{p, q}^{4}\left(p^{-3}+q^{4}\right)-2 q^{3} p^{-2}[m-3]_{p, q}^{4}}{q^{4}\left(R_{p, q}(m, \beta)\right)^{2} T_{p, q}(m, 3)}\right. \\
& +\frac{q^{4}\left(q^{m-3}[3]_{p, q}+p^{3}[m-3]_{p, q}+\beta\right)^{2}\left(q^{m-3}+p[m-3]_{p, q}\right)[m-3]_{p, q}}{q^{4}\left(R_{p, q}(m, \beta)\right)^{2} T_{p, q}(m, 3)}
\end{aligned}
$$




$$
\begin{aligned}
& \left.+\frac{p^{-5}[2]_{p, q}[m]_{p, q}^{3}+2 \alpha q^{2} p^{-3}[m-3]_{p, q}[m]_{p, q}^{2}}{q^{3}\left(R_{p, q}(m, \beta)\right)^{2} T_{p, q}(m, 3)}\right\}\left(x^{2}+x\right) \\
& +\frac{\alpha^{2}}{\left(R_{p, q}(m, \beta)\right)^{2}} .
\end{aligned}
$$

From (4), we have

$$
\begin{aligned}
H_{m, p, q}^{\alpha, \beta}\left((t-x)^{2}, x\right) & \leq\left\{\frac{2\left(1+p^{-2} q^{3}\right)[m-3]_{p, q}^{4}-2 q^{3} p^{-2}[m-3]_{p, q}^{4}}{q^{4}\left(R_{p, q}(m, \beta)\right)^{2} T_{p, q}(m, 3)}\right. \\
& +\frac{\left(q^{m+1} p^{6}+2[3]_{p, q} p^{4} q^{m+1}+2 p^{4} q^{4} \beta+4[3]_{p, q} p^{6} q^{m-3}\right)[m-3]_{p, q}^{3}}{q^{4}\left(R_{p, q}(m, \beta)\right)^{2} T_{p, q}(m, 3)} \\
& +\frac{\left(p q^{4} \beta^{2}+2 \beta p q^{m+1}\left(p^{2}+[3]_{p, q}\right)+2[3]_{p, q} p^{3} q^{2 m-2}\right.}{q^{4}\left(R_{p, q}(m, \beta)\right)^{2} T_{p, q}(m, 3)} \\
& +\frac{\left.[3]_{p, q}^{2} p q^{2 m-2}\left(1+6 p^{3} q^{-4}\right)\right)[m-3]_{p, q}^{2}}{q^{4}\left(R_{p, q}(m, \beta)\right)^{2} T_{p, q}(m, 3)} \\
& +\frac{\left(4[3]_{p, q}^{3} q^{3 m-9}+[3]_{p, q}^{2} q^{3 m-5}+\beta^{2} q^{m+1}+2 \beta q^{2 m-2}[3]_{p, q}\right)[m-3]_{p, q}}{q^{4}\left(R_{p, q}(m, \beta)\right)^{2} T_{p, q}(m, 3)} \\
& \left.+\frac{[3]_{p, q}^{4} p^{-3} q^{4 m-12}+[m]_{p, q}^{3}[2]_{p, q} q p^{-5}+2 \alpha p^{-3} q^{3}[m]_{p, q}^{2}[m-3]_{p, q}}{q^{4}\left(R_{p, q}(m, \beta)\right)^{2} T_{p, q}(m, 3)}\right\}\left(x^{2}+x\right) \\
& +\frac{\alpha^{2}}{\left(R_{p, q}(m, \beta)\right)^{2}} \quad \alpha^{2} \\
& \leq\left(\frac{2\left(1-p^{-2} q^{3}\right)}{q^{4}}+\frac{288(\alpha+\beta+1)^{2}[m]_{p, q}}{q^{4} T_{p, q}(m, 3)}\right)\left(x^{2}+x\right)+\frac{\alpha^{2}}{\left(R_{p, q}(m)\right)^{2}} \\
&
\end{aligned}
$$

And the proof of the Lemma 2 is now completed.

Assume that, $B[0, \infty)$ denotes the set of all bounded functions from $[0, \infty)$ to $\mathbb{R}$. Having the norm $\|g\|_{B}=\sup \{|g(x)|: x \in[0, \infty)\}, B[0, \infty)$ is a normed space. For all continuous functions in $B[0, \infty)$, the subspace is denoted by $C_{B}[0, \infty)$. The first modulus of continuity on finite interval $[0, b], b>0$ is denoted as follows;

$$
w_{[0, b]}(g, \delta)=\sup _{0<h \leq \delta, x \in[0, b]}|g(x+h)-g(x)|
$$

The Peetre's K-functional is defined with the help of the following representation

$$
K_{2}(g, \delta)=\inf \left\{\|g-f\|_{B}+\delta\left\|f^{\prime \prime}\right\|_{B}: f \in W_{\infty}^{2}\right\}, \quad \delta>0
$$

where $W_{\infty}^{2}=\left\{f \in C_{B}[0, \infty): f^{\prime}, f^{\prime \prime} \in C_{B}[0, \infty)\right\}$. There is a positive constant $C$ at Theorem 2.4 on $\mathrm{p} .177$ in Gadzhiev (1976), such that 


$$
K_{2}(g, \delta) \leq C w_{2}(g, \sqrt{\delta})
$$

where

$$
w_{2}(g, \sqrt{\delta})=\sup _{0<h \leq \sqrt{\delta}} \sup _{x \in[0, b]}|g(x+2 h)-g(x+h)-g(x)| .
$$

In Gadzhiev (1976), Gadzhiev proved the weighted Korovkin-type theorems. Let $\sigma(x)=1+x^{2}$.

$B_{\sigma}[0, \infty)$ denotes the set of all functions $g$, from $[0, \infty)$ to $\mathbb{R}$ that meets the growth condition $|g(x)| \leq M_{g} \sigma(x)$.

In this inequality, $M_{g}$ is a constant depending only on $g \cdot B_{\sigma}[0, \infty)$ is a normed space with the norm

$\|g\|_{\sigma}=\sup \left\{\frac{|g(x)|}{\sigma(x)}: x \in[0, \infty)\right\} . C_{\sigma}[0, \infty)$ denotes the subspace of all continuous functions in $B_{\sigma}[0, \infty)$ and $C_{\sigma}^{*}[0, \infty)$ denotes the subspace of all functions $g \in C_{\sigma}[0, \infty)$ whose following limit exists finitely

$$
\lim _{|x| \rightarrow \infty} \frac{|g(x)|}{\sigma(x)} .
$$

Now, the direct results can be given. Because the following lemma is a routine, its proof is omitted.

\section{Lemma 3 Let}

$$
\bar{H}_{m, p, q}^{\alpha, \beta}(g, x)=H_{m, p, q}^{\alpha, \beta}(g, x)-g\left(\frac{p^{-2}[m]_{p, q}^{2}}{q R_{p, q}(m, \beta) T_{p, q}(m, 2)} x+\frac{\alpha}{R_{p, q}(m, \beta)}\right)+g(x) .
$$

For the operators (10), the following equalities are asserted:

(i) $\bar{H}_{m, p, q}^{\alpha, \beta}(1, x)=1$,

(ii) $\bar{H}_{m, p, q}^{\alpha, \beta}(t, x)=x$,

(iii) $\bar{H}_{m, p, q}^{\alpha, \beta}(t-x, x)=0$.

Lemma 4 Let $0<q<p \leq 1$ and $m>3$. Then $g^{\prime \prime} \in C_{B}[0, \infty)$, we have the following inequality

$$
\left|\bar{H}_{m, p, q}^{\alpha, \beta}(g, x)-g(x)\right| \leq \zeta_{m, p, q}^{\alpha, \beta}(x)\left\|g^{\prime \prime}\right\|_{B}
$$

where $\zeta_{m, p, q}^{\alpha, \beta}(x)=\left(\frac{2\left(1-p^{-2} q^{3}\right)}{q^{4}}+\frac{332(\alpha+\beta+1)^{2}}{q^{4} T_{p, q}(m, 2)}\right)\left(x^{2}+x\right)+\frac{\alpha^{2}}{\left(R_{p, q}(m, \beta)\right)^{2}}$.

Proof Using Taylor's expansion

$$
g(t)=g(x)+(t-x) g^{\prime}(x)+\int_{x}^{t}(t-u) g^{\prime \prime}(u) d u
$$

and Lemma 3, we obtain

$$
\bar{H}_{m, p, q}^{\alpha, \beta}(g, x)-g(x)=\bar{H}_{m, p, q}^{\alpha, \beta}\left(\int_{x}^{t}(t-u) g^{\prime \prime}(u) d u, x\right) .
$$


Then, using Lemma1 and the following inequality

$$
\left|\int_{x}^{t}(t-u) g^{\prime \prime}(u) d u\right| \leq\left\|g^{\prime \prime}\right\|_{B} \frac{(t-x)^{2}}{2}
$$

we get

$$
\begin{aligned}
& \left|\bar{H}_{m, p, q}^{\alpha, \beta}(g, x)-g(x)\right| \leq \mid H_{m, p, q}^{\alpha, \beta}\left(\int_{x}^{t}(t-u) g^{\prime \prime}(u) d u, x\right) \\
& -\int_{x}^{\frac{p^{-2}[m]_{p, q}^{2}}{q R_{p, q}(m, \beta) T_{p, q}(m, 2)}} \int^{x+\frac{\alpha}{R_{p, q}(m, \beta)}}\left(\frac{p^{-2}[m]_{p, q}^{2}}{q R_{p, q}(m, \beta) T_{p, q}(m, 2)} x+\frac{\alpha}{R_{p, q}(m, \beta)}-u\right) g^{\prime \prime}(u) d u \\
& \leq \frac{\left\|g^{\prime \prime}\right\|_{B}}{2} H_{m, p, q}^{\alpha, \beta}\left((t-x)^{2}, x\right)+\frac{\left\|g^{\prime \prime}\right\|_{B}}{2}\left(\left(\frac{p^{-2}[m]_{p, q}^{2}}{q R_{p, q}(m, \beta) T_{p, q}(m, 2)}-1\right)+\frac{\alpha}{R_{p, q}(m, \beta)}\right)^{2} \\
& \leq \frac{\left\|g^{\prime \prime}\right\|_{B}}{2}\left\{\left(\frac{2\left(1-p^{-2} q^{3}\right)}{q^{4}}+\frac{288(\alpha+\beta+1)^{2}[m]_{p, q}}{q^{4} T_{p, q}(m, 3)}\right)\left(x^{2}+x\right)+\frac{\alpha^{2}}{\left(R_{p, q}(m, \beta)\right)^{2}}\right\} \\
& +\frac{\left\|g^{\prime \prime}\right\|_{B}}{2}\left\{\left(\frac{p^{-4}[m]_{p, q}^{4}}{\left(q R_{p, q}(m, \beta) T_{p, q}(m, 2)\right)^{2}}-\frac{2 p^{-2} q[m]_{p, q}^{2}\left([m]_{p, q}+\beta\right)[m-2]_{p, q}}{\left(q R_{p, q}(m, \beta) T_{p, q}(m, 2)\right)^{2}}\right.\right. \\
& \left.-\frac{q^{2}\left([m]_{p, q}+\beta\right)^{2}[m-2]_{p, q}^{2}}{\left(q R_{p, q}(m, \beta) T_{p, q}(m, 2)\right)^{2}}\right) x^{2}+\frac{2 \alpha\left(p^{-2}[m]_{p, q}^{2}-q\left([m]_{p, q}+\beta\right)[m-2]_{p, q}\right)}{q R_{p, q}^{2}(m, \beta) T_{p, q}(m, 2)} x \\
& +\frac{\alpha^{2}}{\left(R_{p, q}(m, \beta)\right)^{2}} \\
& \leq\left\{\left(\frac{2\left(1-p^{-2} q^{3}\right)}{q^{4}}+\frac{332(\alpha+\beta+1)^{2}}{q^{4} T_{p, q}(m, 2)}\right)\left(x^{2}+x\right)+\frac{\alpha^{2}}{\left(R_{p, q}(m, \beta)\right)^{2}}\right\}\left\|g^{\prime \prime}\right\|_{B} .
\end{aligned}
$$

Finally, the proof of Lemma 4 is completed.

Theorem 1 Let $\left(p_{m}\right),\left(q_{m}\right) \subset(0,1)$ be two sequences with $0<q_{m}<p_{m} \leq 1$ such that $p_{m} \rightarrow 1, q_{m} \rightarrow 1$ as $m \rightarrow \infty$. Then for every $m>3$ and $g \in C_{B}[0, \infty)$, we have the below inequality

$$
\left|H_{m, p_{m}, q_{m}}^{\alpha, \beta}(g, x)-g(x)\right| \leq 2 C w_{2}\left(g, \sqrt{\zeta_{m, p_{m}, q_{m}}^{\alpha, \beta}(x)}\right)+w\left(g, \eta_{m, p_{m}, q_{m}}^{\alpha, \beta}(x)\right)
$$

Where $\eta_{m, p_{m}, q_{m}}^{\alpha, \beta}(x)=\left(\frac{p_{m}^{-2}[m]_{p_{m}, q_{m}}^{2}}{q_{m} R_{p_{m}, q_{m}}(m, \beta) T_{p_{m}, q_{m}}(m, 2)}-1\right) x+\frac{\alpha}{R_{p_{m}, q_{m}}(m, \beta)}$.

Proof. Based on (10), for any $g \in W_{\infty}^{2}$, we obtain the inequality

$$
\left|H_{m, p_{m}, q_{m}}^{\alpha, \beta}(g, x)-g(x)\right| \leq\left|\bar{H}_{m, p_{m}, q_{m}}^{\alpha, \beta}(g-f, x)-(g-f)(x)+H_{m, p_{m}, q_{m}}^{\alpha, \beta}(f, x)-f(x)\right|
$$




$$
+\left|g\left(\frac{p_{m}{ }^{-2}[m]_{p_{m}, q_{m}}^{2} x}{q_{m} R_{p_{m}, q_{m}}(m, \beta) T_{p_{m}, q_{m}}(m, 2)}+\frac{\alpha}{R_{p_{m}, q_{m}}(m, \beta)}\right)-g(x)\right|
$$

From Lemma 4, we get

$$
\begin{aligned}
\left|H_{m, p_{m}, q_{m}}^{\alpha, \beta}(g, x)-g(x)\right| & \leq 2\|g-f\|_{B}+\zeta_{m, p_{m}, q_{m}}^{\alpha, \beta}(x)\left\|f^{\prime \prime}\right\|_{B} \\
& +\left|g\left(\frac{p_{m}{ }^{-2}[m]_{p_{m}, q_{m}}^{2} x}{q_{m} R_{p_{m}, q_{m}}(m, \beta) T_{p_{m}, q_{m}}(m, 2)}+\frac{\alpha}{R_{p_{m}, q_{m}}(m, \beta)}\right)-g(x)\right| .
\end{aligned}
$$

As a result of the equality (6), we have the inequality

$$
\left|H_{m, p_{m}, q_{m}}^{\alpha, \beta}(g, x)-g(x)\right| \leq 2\|g-f\|_{B}+\zeta_{m, p_{m}, q_{m}}^{\alpha, \beta}(x)\left\|f^{\prime \prime}\right\|_{B}+w\left(g, \eta_{m, p_{m}, q_{m}}^{\alpha, \beta}(x)\right) .
$$

Taking the infimum over $g \in W_{\infty}^{2}$ on the right-hand side of the above inequality and then using the inequality (8), we get the desired result.

Theorem 2 Let $\left(p_{m}\right),\left(q_{m}\right) \subset(0,1)$ be two sequences with $0<q_{m}<p_{m} \leq 1$ such that $p_{m} \rightarrow 1, q_{m} \rightarrow 1$ as $m \rightarrow \infty$. Then $g \in C_{\sigma}^{*}[0, \infty)$, we have

$$
\lim _{m \rightarrow \infty}\left\|H_{m, p_{m}, q_{m}}^{\alpha, \beta}(g, x)-g(x)\right\|_{\sigma}=0 .
$$

Proof. From Lemma 1, it is obvious that $\left\|H_{m, p_{m}, q_{m}}^{\alpha, \beta}\left(e_{0}, x\right)-e_{0}\right\|_{\sigma}=0$. Because

$\left|\frac{p_{m}{ }^{-2}[m]_{p_{m}, q_{m}}^{2} x}{q_{m} R_{p_{m}, q_{m}}(m, \beta) T_{p_{m}, q_{m}}(m, 2)}+\frac{\alpha}{R_{p_{m}, q_{m}}(m, \beta)}-x\right| \leq(x+1) o(1)$ and $\frac{1+x}{1+x^{2}}$ is positive and it is bounded from above for each $x \geq 0$, we get

$$
\left\|H_{m, p_{m}, q_{m}}^{\alpha, \beta}\left(e_{1}, x\right)-e_{1}\right\|_{\sigma} \leq \frac{1+x}{1+x^{2}} o(1)
$$

And then $\lim _{m \rightarrow \infty}\left\|H_{m, p_{m}, q_{m}}^{\alpha, \beta}\left(e_{1}, x\right)-e_{1}(x)\right\|_{\sigma}=0$.

Similarly for every $m>3$, we can write

$$
\begin{aligned}
& \left\|H_{m, p_{m}, q_{m}}^{\alpha, \beta}\left(e_{2}, x\right)-e_{2}(x)\right\|_{\sigma}=\sup _{x \in[0, \infty)}\left\{\frac{p_{m}{ }^{-3}[m]_{p_{m}, q_{m}}^{4} x^{2}}{\frac{q_{m}^{4}\left(R_{p_{m}, q_{m}}(m, \beta)\right)^{2} T_{p_{m}, q_{m}}(m, 3)}{1+x^{2}}}\right. \\
& +\frac{\left\{\frac{p_{m}{ }^{-5}[2]_{p_{m}, q_{m}}[m]_{p_{m}, q_{m}}^{3} x^{2}}{q_{m}^{4}\left(R_{p_{m}, q_{m}}(m, \beta)\right)^{2} T_{p_{m}, q_{m}}(m, 3)}+\frac{2 \alpha p_{m}{ }^{-3}[m]_{p_{m}, q_{m}}^{2}}{q_{m}^{2}\left(R_{p_{m}, q_{m}}(m, \beta)\right)^{2} T_{p_{m}, q_{m}}(m, 3)}\right\} x}{1+x^{2}} \\
& \left.+\frac{\frac{\alpha^{2}}{\left(R_{p_{m}, q_{m}}(m, \beta)\right)^{2}}-x^{2} \mid}{1+x^{2}}\right\}
\end{aligned}
$$




$$
\leq \sup _{x \in[0, \infty)} \frac{1+x+x^{2}}{1+x^{2}} o(1)
$$

and we get $\lim _{m \rightarrow \infty}\left\|H_{m, p_{m}, q_{m}}^{\alpha, \beta}\left(e_{2}, x\right)-e_{2}(x)\right\|_{\sigma}=0$. Therefore, by using A. D. Gadzhiev.s Theorem in Gadzhiev (1976), we obtain Theorem 2's result.

Lemma 5 Let $g \in C_{\sigma}[0, \infty),\left(p_{m}\right),\left(q_{m}\right) \subset(0,1)$ be two sequences with $0<q_{m}<p_{m} \leq 1$ such that $p_{m} \rightarrow$ $1, q_{m} \rightarrow 1$ as $m \rightarrow \infty$ and $w_{[0, d+1]}(g, \delta)$ be its modulus of continuity on the finite interval $[0, d+1] d>0$. Then for every $m>3$, there exists a constant $C>0$ such that the inequality holds

$$
\left\|H_{m, p_{m}, q_{m}}^{\alpha, \beta}(g, x)-g(x)\right\|_{C[0, d]} \leq C\left\{(d+1)^{2} \xi_{m, p_{m}, q_{m}}^{\alpha, \beta}(d)+w_{[0, d+1]}\left(g, \sqrt{\xi_{m, p_{m}, q_{m}}^{\alpha, \beta}(d)}\right)\right\},
$$

where

$$
\xi_{m, p_{m}, q_{m}}^{\alpha, \beta}(d)=\left(\frac{2\left(1-p_{m}^{-2} q_{m}^{3}\right)}{q_{m}^{4}}+\frac{288(\alpha+\beta+1)^{2}[m]_{p_{m}, q_{m}}}{q_{m}^{4} T_{p_{m}, q_{m}}(m, 3)}\right)\left(d^{2}+d\right)+\frac{\alpha^{2}}{\left(R_{p_{m}, q_{m}}(m, \beta)\right)^{2}} .
$$

Proof. Let $x \in[0, d]$ and $t>d+1$. Since $t-x>1$, we have

$$
\begin{aligned}
|g(t)-g(x)| & \leq M_{g}\left(2+t^{2}+x^{2}\right) \\
& \leq 3 M_{g}(1+d)^{2}(t-x)^{2}
\end{aligned}
$$

Let $x \in[0, d]$ and $t<d+1$ and $\delta>0$. Then we have

$$
|g(t)-g(x)| \leq\left(1+\frac{|t-x|}{\delta}\right) w_{[0, d+1]}(g, \delta) .
$$

With the help of (11) and (3.12), we can write

$$
|g(t)-g(x)| \leq 3 M_{g}(1+d)^{2}(t-x)^{2}+\left(1+\frac{|t-x|}{\delta}\right) w_{[0, d+1]}(g, \delta) .
$$

Then, using Lemma 2 and Cauchy-Schwarz's inequality, we get the following inequalities

$$
\begin{aligned}
\left|H_{m, p_{m}, q_{m}}^{\alpha, \beta}(g, x)-g(x)\right| & \leq 3 M_{g}(1+d)^{2} H_{m, p_{m}, q_{m}}^{\alpha, \beta}\left((t-x)^{2}, x\right) \\
& +w_{[0, d+1]}(g, \delta)\left[1+\frac{1}{\delta}\left(H_{m, p_{m}, q_{m}}^{\alpha, \beta}\left((t-x)^{2}, x\right)\right)^{1 / 2}\right] \\
& \leq 3 M_{g}(1+d)^{2} \xi_{m, p_{m}, q_{m}}^{\alpha, \beta}(x)+w_{[0, d+1]}(g, \delta)\left[1+\frac{1}{\delta}\left(\xi_{m, p_{m}, q_{m}}^{\alpha, \beta}(x)\right)^{1 / 2}\right],
\end{aligned}
$$

where

$$
\xi_{m, p_{m}, q_{m}}^{\alpha, \beta}(x)=\left(\frac{2\left(1-p_{m}^{-2} q_{m}^{3}\right)}{q_{m}^{4}}+\frac{288(\alpha+\beta+1)^{2}[m]_{p_{m}, q_{m}}}{q_{m}^{4} T_{p_{m}, q_{m}}(m, 3)}\right)\left(x^{2}+x\right)+\frac{\alpha^{2}}{\left(R_{p_{m}, q_{m}}(m, \beta)\right)^{2}}
$$

Setting 


$$
\delta^{2}:=\xi_{m, p_{m}, q_{m}}^{\alpha, \beta}(d)=\left(\frac{2\left(1-p_{m}^{-2} q_{m}^{3}\right)}{q_{m}^{4}}+\frac{288(\alpha+\beta+1)^{2}[m]_{p_{m}, q_{m}}}{q_{m}^{4} T_{p_{m}, q_{m}}(m, 3)}\right)\left(d^{2}+d\right)+\frac{\alpha^{2}}{\left(R_{p_{m}, q_{m}}(m, \beta)\right)^{2}}
$$

and $C=\min \left\{3 M_{g}, 2\right\}$. Therefore, the proof of Lemma 5 is finished.

Theorem 3 Let $\lambda>0,\left(p_{m}\right),\left(q_{m}\right) \subset(0,1)$ be two sequences with $0<q_{m}<p_{m} \leq 1$ such that $p_{m} \rightarrow 1$, $q_{m} \rightarrow 1$ as $m \rightarrow \infty$ and $g \in C_{\sigma}^{*}[0, \infty)$. Then we have

$$
\lim _{m \rightarrow \infty} \sup _{x \geq 0} \frac{\left|H_{m, p_{m}, q_{m}}^{\alpha, \beta}(g, x)-g(x)\right|}{1+x^{2+\lambda}}=0 .
$$

Proof. For $\lambda>0, g \in C_{\sigma}^{*}[0, \infty)$ and $b>1$, the following inequality is ensured

$$
\begin{aligned}
\sup _{x \geq 0} \frac{\left|H_{m, p_{m}, q_{m}}^{\alpha, \beta}(g, x)-g(x)\right|}{1+x^{2+\lambda}} & \leq \sup _{0 \leq x<d} \frac{\left|H_{m, p_{m}, q_{m}}^{\alpha, \beta}(g, x)-g(x)\right|}{1+x^{2+\lambda}}+\sup _{d \leq x} \frac{\left|H_{m, p_{m}, q_{m}}^{\alpha, \beta}(g, x)-g(x)\right|}{1+x^{2+\lambda}} \\
& \leq\left\|H_{m, p_{m}, q_{m}}^{\alpha, \beta}(g, x)-g(x)\right\|_{C[0, d]}+\sup _{d \leq x} \frac{\left|H_{m, p_{m}, q_{m}}^{\alpha, \beta}(g, x)-g(x)\right|}{1+x^{2}} \\
& \leq\left\|H_{m, p_{m}, q_{m}}^{\alpha, \beta}(g, x)-g(x)\right\|_{C[0, d]}+\left\|H_{m, p_{m}, q_{m}}^{\alpha, \beta}(g, x)-g(x)\right\|_{\sigma}
\end{aligned}
$$

Using Lemma 5 and Theorem 2, the proof of Theorem 3 is provided.

\section{CONFLICT OF INTEREST}

The authors declare no conflict of interest.

\section{REFERENCES}

Acar, T., Aral, A., \& Mohiuddine, S. A. (2016). On Kantorovich modication of ( $p, q)$-Baskakov operators. Journal of Inequalities and Applications, 2016, 98. doi:10.1186/s13660-016-1045-9

Acar, T., Aral A., \& Mohiuddine, S. A. (2018). Approximation by Bivariate $(p, q)$-Bernstein-Kantorovich operators. Iranian Journal of Science and Technology, Transactions A: Science, 42(2), 655-662. doi:10.1007/s40995-016-0045-4

Aral, A., Gupta, V., \& Agarwal, R. P. (2013). Applications of q-calculus in operator theory. Springer, New York. doi:10.1007/978-1-4614-6946-9

Cai, Q-B., Sofyalıoglu, M., Kanat, K., \& Çekim, B. (2021). Some approximation results for the new modification of Bernstein-Beta operators AIMS Mathematics, 7(2), 1831-1844. doi:10.3934/math.2022105

De Sole, A., \& Kac, V. G. (2005). On integral representations of q-gamma and q-beta functions. Atti Accad. Naz. Lincei Cl. Sci. Fis. Mat. Natur. Rend. Lincei Mat. Appl., 16(1), 11-29.

Dinlemez, Ü., Yüksel, İ., \& Altın, B. (2014). A note on the approximation by the q-hybrid summation integral type operators. Taiwanese Journal of Mathematics, 18(3), 781-792.

Doğru, O., \& Gupta, V. (2005). Monotonicity and the asymptotic estimate of Bleimann Butzer and Hahn operators based on q -integers. Georgian Mathematical Journal, 12(3), 415-422.

Doğru, O., \& Gupta, V. (2006). Korovkin-type approximation properties of bivariate q-Meyer-König and Zeller operators. Calcolo, 43(1), 51-63. doi:10.1007/s10092-006-0114-8

Gadzhiev, A. D. (1976). Theorems of the type of P. P. Korovkin type theorems. Matematicheskie Zametki, 20(5), 781-786. English Translation: Math. Notes, 20(5/6), 996-998.

Gupta, V., \& Heping, W. (2008). The rate of convergence of q-Durrmeyer operators for $0<\mathrm{q}<1$. Mathematical Methods in the Applied Sciences, 31(16), 1946-1955. doi:10.1002/mma.1012 
Gupta, V., \& Aral, A. (2010). Convergence of the q-analogue of Szász-beta operators. Applied Mathematics and Computation, 216(2), 374-380. doi:10.1016/j.amc.2010.01.018

Gupta, V., \& Karsli, H. (2012). Some approximation properties by q-Szász-Mirakyan-Baskakov-Stancu operators. Lobachevskii Journal of Mathematics, 33(2), 175-182. doi:10.1134/S1995080212020138

Gupta, V. (2018). (p, q)-Szász-Mirakyan-Baskakov Operators. Complex Analysis and Operator Theory, 12 , 17-25. doi:10.1007/s11785-015-0521-4

Jackson, F. H. (1910). On q-Definite Integrals. The Quarterly Journal of Pure and Applied Mathematics, 41(15), 193-203.

Kac, V. G., \& Cheung, P. (2002). Quantum Calculus. Part of the Universitext book series, Springer-Verlag, New York. doi:10.1007/978-1-4613-0071-7

Kanat, K., \& Sofyalıoğlu, M. (2018). Approximation by $(p, q)$-Lupaş-Schurer-Kantorovich operators. Journal of Inequalities and Applications, 2018, 263. doi:10.1186/s13660-018-1858-9

Kanat, K., \& Sofyalıŏlu, M. (2021). On Stancu type Szász-Mirakyan-Durrmeyer Operators Preserving $\exp (2 \mathrm{ax})$, a > 0. Gazi University Journal of Science, 34(1), 196-209. doi:10.35378/gujs.691419

Koelink, H. T., \& Koornwinder, T. H. (1990). q-special functions, a tutorial. In: M. Gerstenhaber, \& J. Stasheff (Eds.) Deformation Theory and Quantum Groups with Applications to Mathematical Physics (Proceedings of an AMS-IMS-SIAM 1990), Contemporary Mathematics, 134, 141-142.

Lupaş, A. (1987). A q-analogue of the Bernstein operator. In: Seminar on numerical and statistical calculus, University of Cluj-Napoca, 9, 85-92.

Mursaleen, M., Ansari, K. J., \& Khan, A. (2015a). On (p, q)-analogue of Bernstein operators. Applied Mathematics and Computation, 266, 874-882. doi:10.1016/j.amc.2015.04.090

Mursaleen, M., Ansari, K. J., \& Khan, A. (2015b). Some Approximation Results by ( $p$, $q$ )-analogue of Bernstein-Stancu operators. Applied Mathematics and Computation, 264, 392-402. doi:10.1016/j.amc.2015.03.135

Mursaleen, M., Nasiuzzaman, Md., \& Nurgali, A. (2015c). Some approximation results on Bernstein-Schurer operators dened by $(p, q)$-integers. Journal of Inequalities and Applications, 2015, 249. doi:10.1186/s13660$\underline{015-0767-4}$

Phillips, G. M. (1997). Bernstein polynomials based on the q-integers. Annals of Numerical Mathematics, 4(14), 511-518.

Sahai, V., \& Yadav, S. (2007). Representations of two parameter quantum algebras and ( $p$, $q$ )-special functions. Journal of Mathematical Analysis and Applications, 335(1), 268-279. doi:10.1016/j.jmaa.2007.01.072

Sofyalığlu, M., Kanat, K., \& Çekim, B. (2021). Parametric generalization of the Meyer-König-Zeller operators. Chaos, Solitons \& Fractals, 152, 111417. doi:10.1016/j.chaos.2021.111417

Yüksel, İ. (2013). Direct results on the q-mixed summation integral type operators. J. Applied Functional Analysis, 8(2), 235-245. 